\title{
Magnetic-field-induced phase transitions in the quasi-one-dimensional organic conductor HMTSF-TCNQ
}

\author{
K. Murata ${ }^{1}$, Y. Fukumoto ${ }^{1}$, K. Yokogawa ${ }^{1}$, R. Takaoka ${ }^{1}$, W. Kang $^{2}$, J.S. Brooks ${ }^{3}$, D. Graf ${ }^{3}$, \\ H. Yoshino ${ }^{1}$, T. Sasaki ${ }^{4}$, and R. Kato ${ }^{5}$ \\ E-mail: muratak@sci.osaka-cu.ac.jp \\ ${ }^{1}$ Graduate School of Science, Osaka City University, Osaka 558-8585, Japan \\ ${ }^{2}$ Ewha Womans University, Seoul 120-750, Korea \\ ${ }^{3}$ Nat'l. High Mag. Field Lab. Florida St. University, Tallahassee FL 32306-4005, USA \\ ${ }^{4}$ Institute for Materials Research, Tohoku University, Sendai 980-8577, Japan \\ ${ }^{5}$ RIKEN, Wako, Saitama 351-0198
}

Received November 5, 2013

\begin{abstract}
Motivated by an interest to see if the field-induced (FI) phase in the charge-density wave (CDW) system is similar to the field-induced-SDW (FISDW) in (TMTSF) ${ }_{2} \mathrm{X}$, (TMTSF: tetramethyltetraselenafulvalene), we examined the magnetic-field-induced phases in a quasi-one-dimensional (Q1D) organic conductor HMTSF-TCNQ (hexamethylene-tetraselenafulvalene-tetracyanoquinodimethane) under a pressure of $1.1 \mathrm{GPa}$, where the CDW occurring at $30 \mathrm{~K}$ is suppressed. The work was carried out by measurements of angular-dependent magnetoresistance oscillations and exploratory work on the Hall effect. It turned out that the FI-phase, most likely a FICDW for $B>0.1 \mathrm{~T}$, accompany a quantum Hall effect, and the FI-phase transitions are controlled by the field component along the least conducting axis. Above $10 \mathrm{~T}$, the lowest Landau level of the small 2D Fermi pocket (due to incomplete nesting of Fermi surface) exceeds the Fermi level, reaching the quantum limit. Although there are many differences between the CDW (HMTSF-TCNQ) and SDW ((TMTSF) 2 X) systems, a similar scenario for field-induced phases seems to hold.
\end{abstract}

PACS: 73.43.-f Quantum Hall effects;

74.70.Kn Organic superconductors;

71.45.Lr Charge-density-wave systems;

75.30.Fv Spin-density waves.

Keywords: HMTSF-TCNQ, charge-density wave, magnetoresistance, field-induced phase transitions, Fermi surface, nesting.

\section{Introduction}

The first organic superconductor (TMTSF) ${ }_{2} \mathrm{PF}_{6}$ shows a spin-density wave (SDW) at $12 \mathrm{~K}$ at ambient pressure. When the SDW is suppressed by a pressure of $0.65 \mathrm{GPa}$, superconductivity appears [1]. The suppression of SDW is understood as a recovery of the Fermi surface by denesting with increasing pressure. Then by applying a magnetic field superconductivity is suppressed, and successive magnetic-field-induced SDW (FISDW) transitions appear, schematically shown in Fig. 1. The FISDW is interpreted as the recovery of nesting due to one-dimensionalization by a strong magnetic field. But this nesting is imperfect, and a resultant small 2D Fermi pocket provides a cascade of phase transitions. This material drew further attention by the fact that the FISDW states produce a new type of quantum Hall effect (QHE) [2] quite different from the conventional QHE seen in GaAlAs.

It is of great interest to see if the field-induced phase scenario holds in the charge-density wave (CDW) case. For this purpose, we studied the quasi-one-dimensional (Q1D) organic conductor, HMTSF-TCNQ, shown in Fig. 2. The $\mathrm{CDW}\left(T_{C D W}=30 \mathrm{~K}\right.$ at ambient pressure [3-5]) of this crystal is relatively easily suppressed by the pressure of $1.1 \mathrm{GPa}$, as shown in Figs. 3-4, in agreement with previous work [6]. (We note that for TTF-TCNQ $10 \mathrm{GPa}$ are required to suppress the CDW phase [7].) Hence with HMTSF-TCNQ, it is easy to use a simple pressure clamp apparatus in high magnetic fields. Our studies on HMTSF-TCNQ show magneto- 


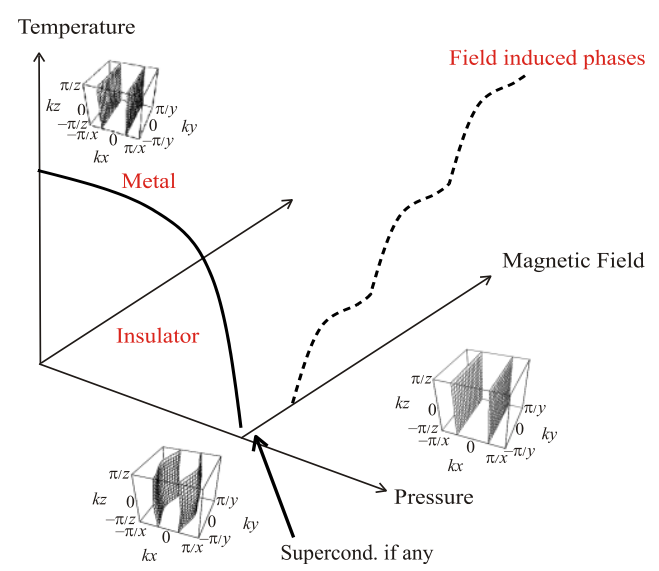

Fig. 1. (Color online) Schematic diagram of a 1D conductor. Insulating phase is either SDW or CDW. The images show pairs of $1 \mathrm{D}$ Fermi surfaces in the 1st. In the case of HMTSF-TCNQ, there are both electron and Brillouin zone hole Fermi surface pairs in the 1st Brillouin zone. Orientation of the magnetic field is parallel to the least conducting axis.

resistance kinks around $1.1 \mathrm{GPa}$, but neither at ambient pressure nor at $2 \mathrm{GPa}$ [8-12]. This feature implies that a scenario similar to that of FISDW holds.

It should be noted that previously it was difficult to obtain high quality crystals of HMTSF-TCNQ, since most of the samples consisted of many small, oriented crystallites mimicking a single crystal morphology. Such crystals had broad Bragg spots, and the signature of the CDW transition in the resistivity was neither sharp nor large. More recently, one of authors $(\mathrm{R} / \mathrm{K})$ succeeded in synthesizing high quality single crystals, and our understanding of the fieldinduced phase has very much advanced as a result. The purpose of this paper is to present the new data, and to discuss the field-induced phase of this material.

A field-induced CDW in a two dimensional conductor was discussed previously for $\alpha$-(BEDT-TTF $)_{2} \mathrm{KHg}(\mathrm{SCN})_{4}$ and $\alpha$-(BEDT-TTF) $)_{2} \mathrm{TlHg}(\mathrm{SCN})_{4}[13,14]$, where 1D and 2D Fermi surfaces are mixed even below TCDW where the

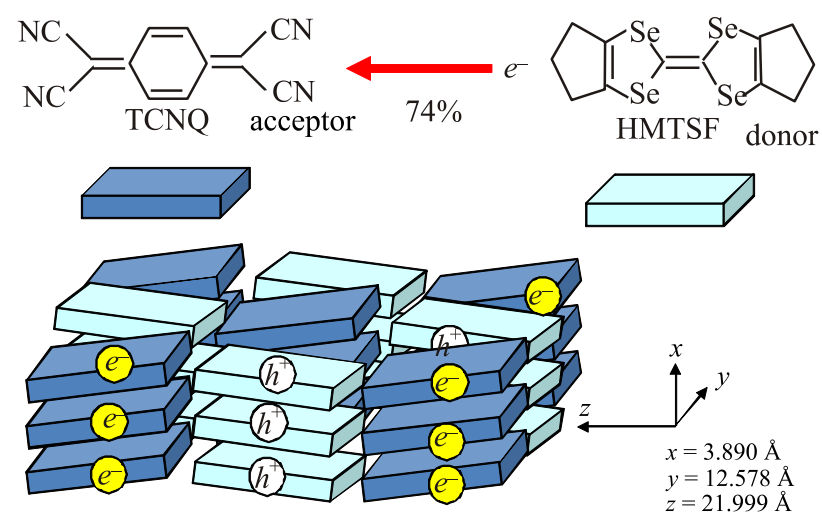

Fig. 2. (Color online) Crystal model of HMTSF-TCNQ.

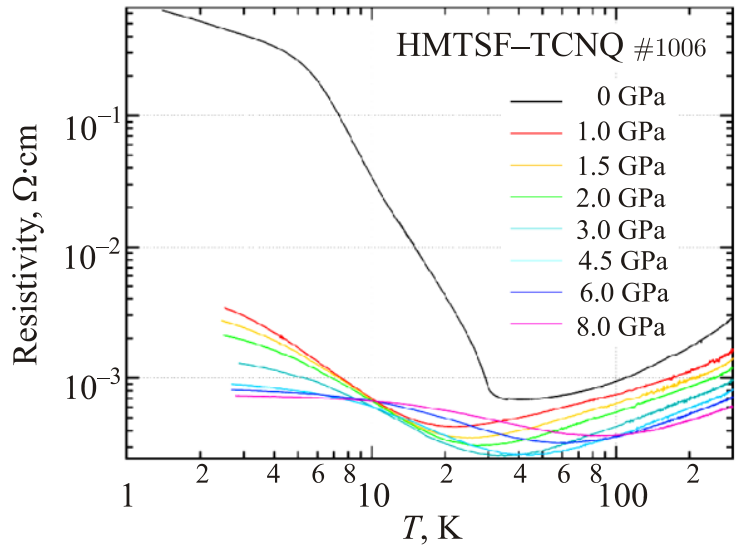

Fig. 3. (Color online) Temperature dependence of resistivity under various pressures studied with cubic anvil pressure apparatus with Daphne 7373.

Fermi-surface reconstructs. In the present case, field-induced phases are indicated by the clear presence of 1D Fermi surfaces and 2D straw-shaped Fermi surfaces.

\section{Experiment}

The crystals of HMTSF-TCNQ were obtained by a diffusion method at a temperature of $40{ }^{\circ} \mathrm{C}$ which resulted in an improvement of the crystal quality. All the samples used for this transport study were examined by x-ray, confirming sharp Brag spots. According to Phillips [3], the crystal is monoclinic, though nearly orthorhombic; $a=$ $=21.999(14), b=12.573(8), c=3.890(1) \AA ; \beta=90.29(4)$. Since previously crystal axes have been assigned in different ways $[3,15]$, to avoid confusion, we adopt the $x, y$, and $z$ labeling of the most, second and least conducting axes respectively, which correspond to the $b, a$, and $c$ (or $c, b$, and a) axes in the Weger's (or Phillips's ) convention.

The sample sizes were \#1201-01 $0.87 \mathrm{~mm} \times 0.14 \mathrm{~mm} \times$ $\times 0.06 \mathrm{~mm}$ and \#1204-01 $0.92 \mathrm{~mm} \times 0.26 \mathrm{~mm} \times 0.13 \mathrm{~mm}$.

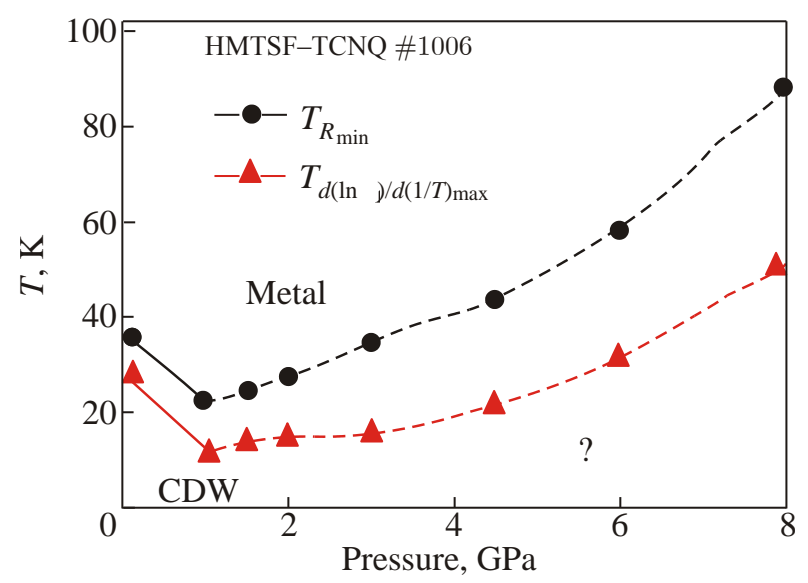

Fig. 4. (Color online) Temperature-pressure phase diagram of HMTSF-TCNQ. 

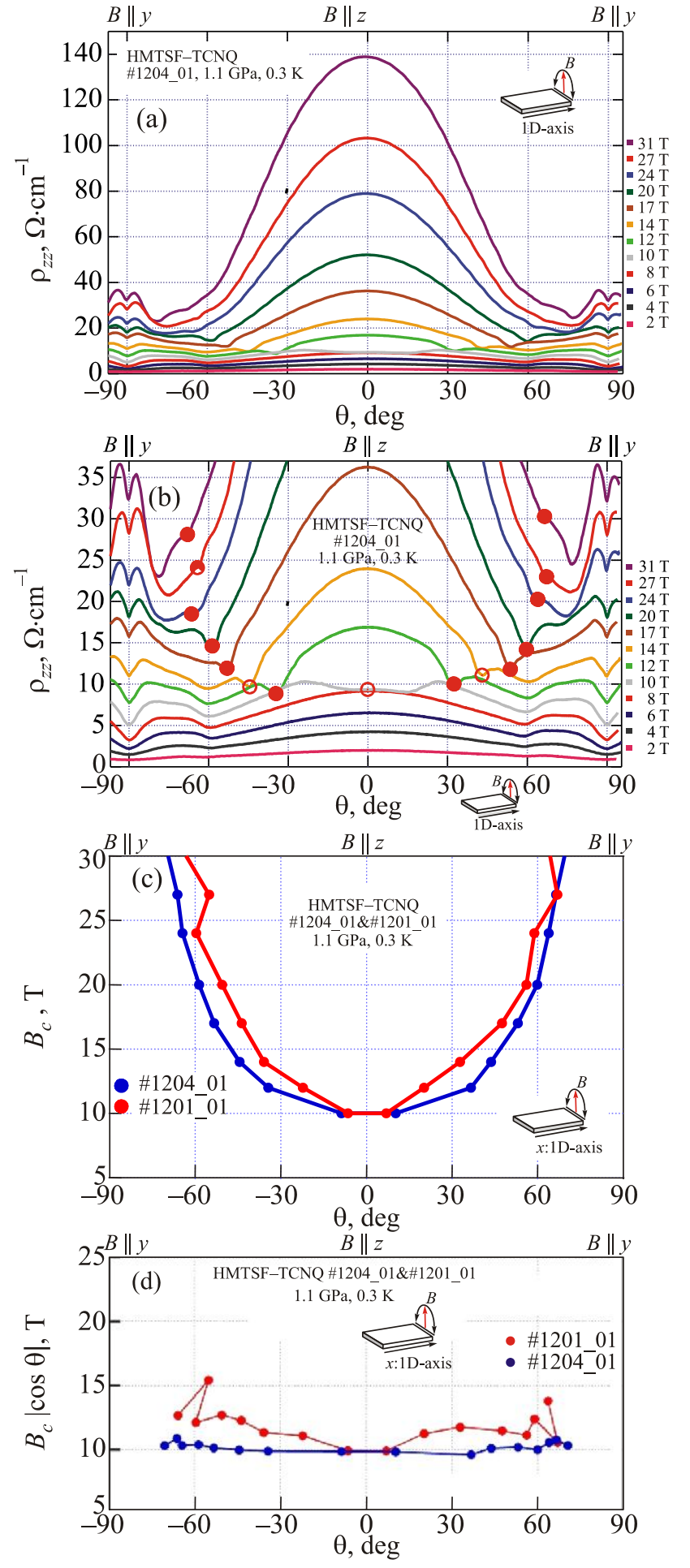

Fig. 5. (Color online). Angular dependence of magnetoresistance oscillation of $\rho_{z z}$ of HMTSF-TCNQ in various fields in the $z-y$ plane at $1.1 \mathrm{GPa}, 0.3 \mathrm{~K}$ (a). Detail of (a), which demonstrates the fine structure of the AMRO and the boundary into the field-induced high resistance phase. The curves are shifted to show the shape of AMRO for increasing fields (b). The field values in (b) are plotted against the field angle in the $z-y$ plane (c). The $z$-component of the field values in (c) are plotted against the field angle in the $z-y$ plane. It is obvious that the clear field-induced phase is occurring by the $z$-component of the field value, which is $10 \mathrm{~T}(\mathrm{~d})$.
Electrical contacts were attached by carbon paste and gold wires 10 or $20 \mu \mathrm{m}$ in diameter. Two samples of HMTSFTCNQ, along with $\mathrm{Sn}$ and manganin pressure monitors were mounted together in a miniature pressure clamp cell, which allows rotation in the cryostat in a high field magnet. The pressure medium was Daphne 7373 [16,17], and pressure at low temperature was determined with the superconducting $T_{c}$ of $\mathrm{Sn}$. Most of the AMRO experiments reported here were performed at $1.1 \mathrm{GPa}$.

We carried out two types of measurements: angular dependence of the magnetoresistance oscillations (AMRO) and the magnetoresistance at fixed temperatures. AMRO is a powerful tool to determine Fermi surface topologies in 1D and 2D metals. The transport lead configuration used for most studies was for the $R_{z z}$ (or $\rho_{z z}$ ) crystallographic direction, with two pairs of contacts on both sides of $x-y$ plane for current and voltage (inset of Fig. 5 (a)). This configuration is suitable when $\rho_{z z}$ is large enough compared with $\rho_{x x}$ and $\rho_{y y}$. We found that $\rho_{z z} / \rho_{x x} \sim 100(1.5 \mathrm{~K})$ and $\sim 80(300 \mathrm{~K})$, which validates the lead configuration.

\section{Boundary of field-induced phase transition observed by AMRO}

Figure 5(a) shows the AMRO of $\rho_{z z}$ with magnetic field rotated in the $z-y$ plane taken in various magnetic fields at $0.35 \mathrm{~K}$. This AMRO configuration yields Lebed-Osada oscillations in the (TMTSF) ${ }_{2} \mathrm{X}$ salts $[18,19]$. It is seen that in HMTSF-TCNQ there are regions where $\rho_{z z}$ is extremely high or low with a clear boundary in between, indicated by symbols in Fig. 5(b). The field values of the symbols are plotted against the field angle in the $z-y$ plane as shown in Fig. 5(c). The $z$-component $B \cos (\theta)$ of the field values in Fig. 5(c) are plotted against the field angle in the $z-y$ plane as shown in Fig. 5(d). It is quite obvious that the field induced phase is determined by the $z$-component $(\sim 10 \mathrm{~T})$ of the field values.

Similar AMRO measurements in the $z-x$ plane are shown in Fig. 6(a) in various magnetic fields at $0.35 \mathrm{~K}$. In this configuration the AMRO gives the Danner-KangChaikin oscillations in the (TMTSF) $)_{2} \mathrm{X}$ salts [20,21]. A similar description is suitable for Fig. 6(a) through (d). It is seen that there are regions where $\rho_{z z}$ is extremely high or low with a clear boundary between the two, which is shown with symbols in Fig. 6(b). The field values of the symbols are plotted against the field angle in the $z-x$ plane as shown in Fig. 6(c). The $z$-components of the field values plotted against the field angle in the $z-x$ plane are shown in Fig. 6(d). It is again quite obvious that the $z$-component (of $10 \mathrm{~T}$ ) of the field is responsible for the field induced phase.

We can conclude from Fig. 5(d) and Fig. 6(d) that the field-induced high resistance phase is triggered by an orbital effect occurring in the conducting $x-y$ plane. 

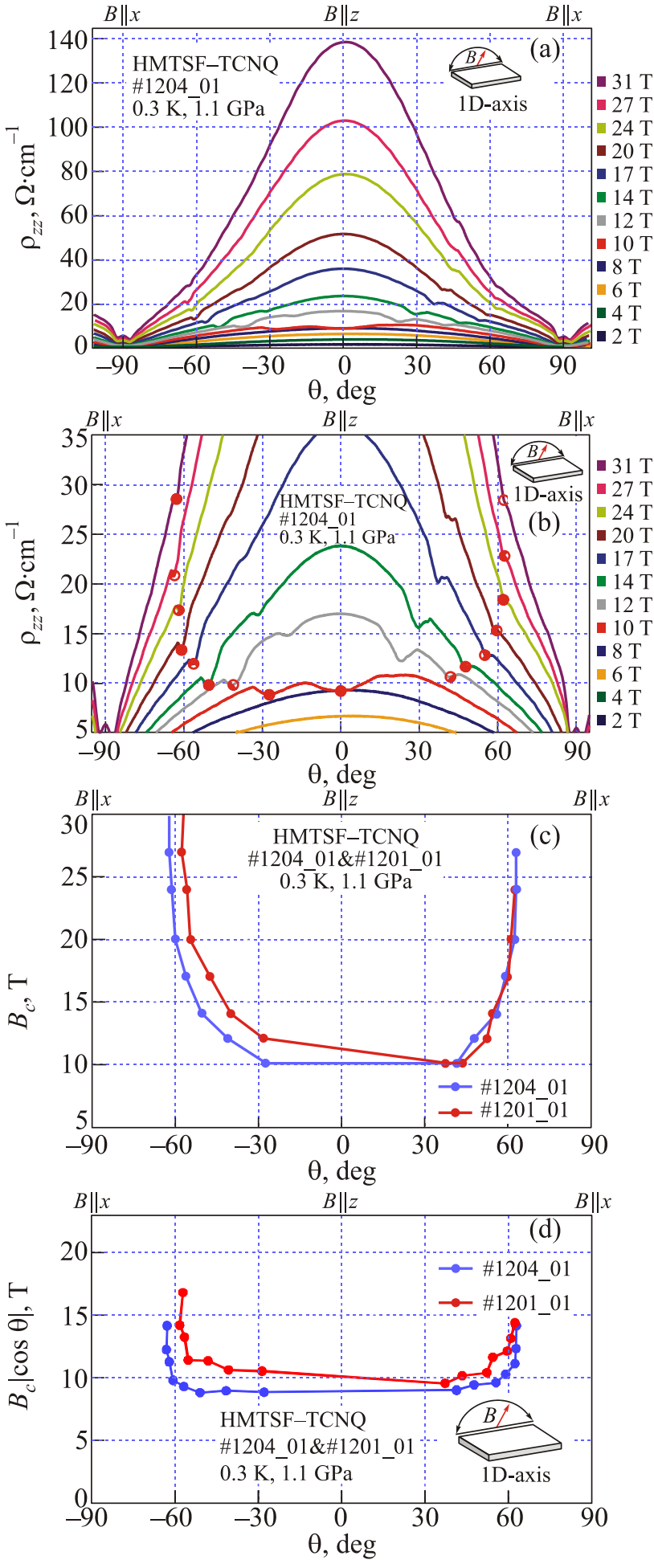

Fig. 6. (Color online). Angular dependence of magnetoresistance oscillation of $\rho_{z z}$ of HMTSF-TCNQ in various fields in the $z-x$ plane at $1.1 \mathrm{GPa}, 0.3 \mathrm{~K}$ (a). Detal of (a), which demonstrates the boundary into the field-induced phase and the fine structure of AMRO. The curves are shifted to show the shape of AMRO with increasing fields (b). The field values of the symbols in (b) are plotted against the field angle in the $z-x$ plane (c). The $z$-component of the field values in (c) are plotted against the field angle in the $z-x$ plane. It is clear that a field-induced phase is occurring due to the $z$-component of the field value, which is $10 \mathrm{~T}$.

\section{Fermi surfaces in and out of field-induced 10 T-phase transition seen by AMRO}

Coming back to Figs. 5(a) and (b), there are many fine oscillations in AMRO. To extract these oscillations from the background, the second derivative of $\rho_{z z}, d^{2} \rho_{z z} / d \theta^{2}$ as a function of angle $\theta$, in the $z-y$ plane is shown in Fig. 7(a). It turned out that $d^{2} \rho_{z z} / d \theta^{2}$ is periodic with $\theta$ even at different magnetic field strengths. It has been discussed in (TMTSF) $)_{2} \mathrm{X}$ salt that this kind of oscillations reflects the undulation of the 1D Fermi surface sheet, and this kind of AMRO shows Lebed-Osada oscillations $[18,19]$. When the magnetic field is applied parallel to the 1D Fermi surface sheet, i.e. perpendicular to the 1D axis, charge carriers make a line shape trajectory in the $k_{y}-k_{z}$ plane of the 1D Fermi surface sheet that is perpendicular to the magnetic field. If the tilt of the trajectory of the electron or hole just matches the ratio of Brillouin zone size of $(2 \pi / y):(2 \pi / z)=z / y$, or a multiple by a rational number, the trajectory in the $k_{y}-k_{z}$ plane draws a repeated path, resulting in non-cancellation of the $v_{z}$-component, and therefore a dip of $\rho_{z z}$ in AMRO. Taking into account this scenario, typical points (humps) in the oscillations in Fig. 7(a) are shown in Fig. 7(b). This oscillatory structure seems to be common in all fields, and hence depends only on the ratio of the lattice constants $z / y=1.59$ at $0.3 \mathrm{~K}$ at $1.1 \mathrm{GPa}$, which can be compared with $z / y=21.999 / 12.573=1.750$ from $x$-ray data at $300 \mathrm{~K}$ at ambient pressure. Differences in the $z / y$ ratio may be due to anisotropic compressibility, a common feature of organic charge transfer salts such as (TMTSF) $)_{2} \mathrm{X}$ [22] and (BEDT-TTF) $)_{2} \mathrm{X}$-salts [23], where the most compressible direction is along the stacking axis ( $x$-axis), and the least is along the molecular axis (z-axis). However, considering that our rotation is not ideally in the $z-y$ plane, compressibility effects are uncertain at present.

Each curve in Fig. 7(a) is separated in Fig. 7(c) by shifting vertically by arbitrary value. It is remarkable that the rapid oscillations of AMRO are pronounced in the region of the phase where $B_{z}>10 \mathrm{~T}$. However, by amplifying the oscillatory behavior for $B_{z}<10 \mathrm{~T}$, as shown in Fig. 7(d), oscillations with similar frequency can be observed. This result indicates that 1D Fermi surface, which is either a pair of electron or hole sheets, is present below and above $B_{z}=10 \mathrm{~T}$. We speculate that above $10 \mathrm{~T}$, only a single pair of 1D Fermi surfaces dominates the behavior, since the oscillations do not change form.

Figure 8 shows the magnetoresistance $\rho_{z z}$ vs $B_{z}=B \cos \theta$ for temperatures from $0.4 \mathrm{~K}$ to $4.2 \mathrm{~K}$. The transition field in $B_{z}$ located at $10 \mathrm{~T}$ at $0.4 \mathrm{~K}$ is found to be unchanged in this temperature range. Slight differences of the transition field from $10 \mathrm{~T}$ are caused by uncertainties in the alignment of the magnetic field. Although there is certainly a 1D Fermi surface, a very high magnetoresistance state appears above $B_{z}=$ $=10 \mathrm{~T}$, with no appreciable structure up to $45 \mathrm{~T}$. It means that by sweeping up the field, $10 \mathrm{~T}$ is the field above which 


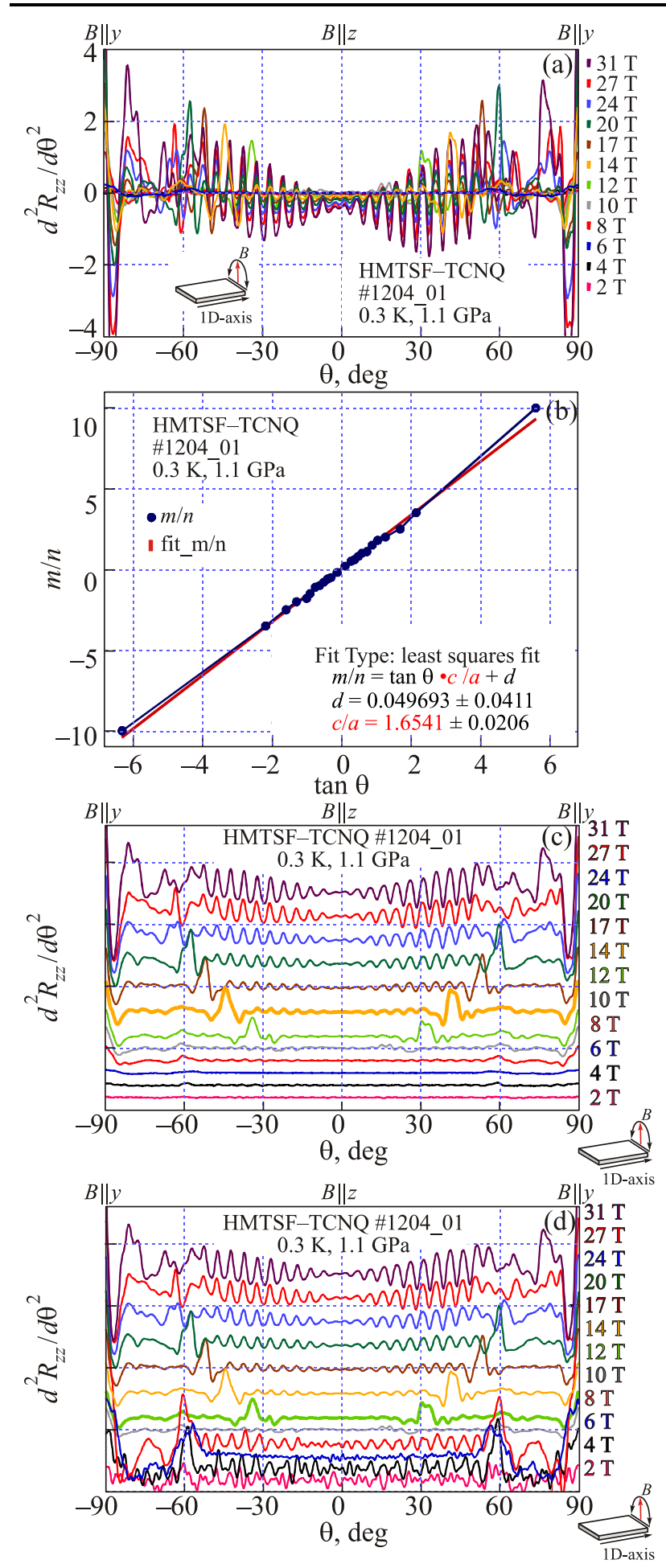

Fig. 7. (Color online). Second derivative of $R_{z z}, d^{2} R_{z z} / d \theta^{2}$, as a function of angle, $\theta$, in the $z-y$ plane (a). Rational number as a function of $\tan \theta$ (b). Data in Fig. 7(a) shifted vertically to show individual curves (c). Expanded view of low-field oscillations in Fig. 7(c) for $B_{z}<10 \mathrm{~T}$. Oscillation amplitude is multiplied by 10 for the data in $8 \mathrm{~T}$ and by 20 in 6,4 , and $2 \mathrm{~T}$. Almost the same oscillations are seen as those for $B_{z}>10 \mathrm{~T}(\mathrm{~d})$.

no transition is expected. The most probable candidate is the Landau quantum limit. The lowest Landau level correspond-
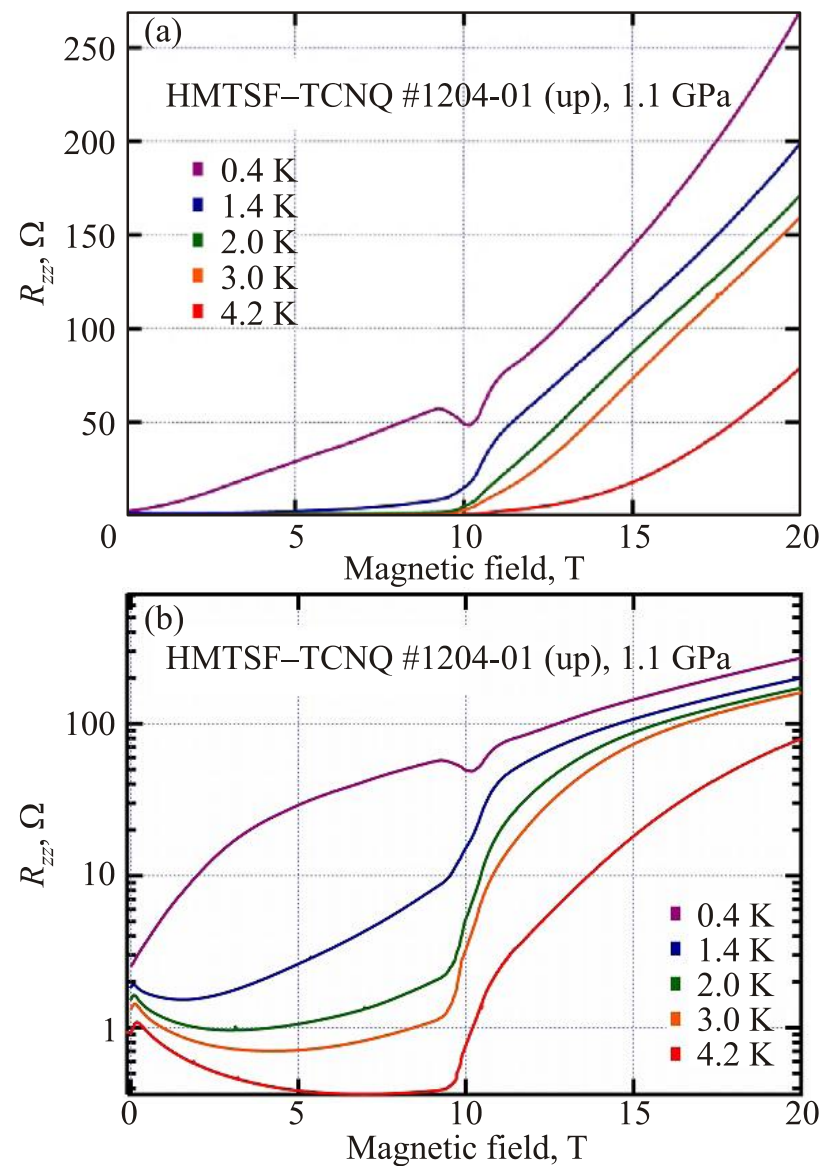

Fig. 8. (Color online). Magnetoresistance in magnetic field of $B_{z}$ in linear scale (a) and logarithmic scale (b).

ing to $10 \mathrm{~T}$ simply accounts for the cross sectional area of the straw shaped Fermi surface through the relation,

$$
S=\frac{\pi e B}{\hbar c}=4.79 \cdot 10^{12} \mathrm{~cm}^{-2} .
$$

This value amounts to $2.33 \%$ of the first Brillouin zone in $k_{x}-k_{y}$ plane, which is $2.04 \cdot 10^{14} \mathrm{~cm}^{-2}$.

In low field, it is also noted that magnetoresistance turns from positive to negative around $0.1 \mathrm{~T}$, which is pronounced at higher temperature towards $4.2 \mathrm{~K}$.

Hall resistance $R_{x y}$ with field direction along the $z$-axis was taken at $1.1 \mathrm{GPa}$ at temperatures between $0.4 \mathrm{~K}$ and $4.2 \mathrm{~K}$. Here a brief description of the Hall effect results is given. Details will be presented in a forthcoming paper after analysis is complete. The value of $R_{x y}$ is negative and linear in $B_{z}$ up to 0.1 or $0.2 \mathrm{~T}$, and is almost constant between 0.2 and $10 \mathrm{~T}$, and finally $R_{x y}$ shows slightly negative slope, and then turns to show a positive slope which continues up to at least $31 \mathrm{~T}$, the maximum field of these measurements. The constant $R_{x y}$ behavior between 0.2 and $10 \mathrm{~T}$ strongly suggests that the quantum Hall effect similar to (TMTSF) ${ }_{2} \mathrm{X}$ occurs. This tendency is approximately the same at temperatures between $0.4 \mathrm{~K}$ and $4.2 \mathrm{~K}$. 


\section{Discussion and summary}

Based on the AMRO, magnetoresistance and some preliminary work on Hall effect (not shown here) under a pressure of $1.1 \mathrm{GPa}$, we can describe, along with some level of speculation, the behavior of the field-induced phases in HMTSF-TCNQ as follows.

In region $\mathrm{I}\left(B_{z}<0.1 \mathrm{~T}\right)$, the Hall resistance $R_{x y}$ is negative and is linear in $B_{z}$, which is consistent with metallic behavior and a constant Hall coefficient. In this region, the magnetoresistance $\rho_{z z}$ is positive at higher temperature up to the measured temperature of $4.2 \mathrm{~K}$, and is positive at low temperature down to at least $0.3 \mathrm{~K}$. In this region, there must be essentially two pairs of Q1D Fermi surface sheets, one with electrons and the other with holes similar to the metallic phase above $T_{C D W}(30 \mathrm{~K})$ at ambient pressure.

In region II $\left(0.1 \mathrm{~T}<B_{z}<10 \mathrm{~T}\right)$, one of the Fermi surface is nested by one-dimensionalization by magnetic field, leaving straw-shaped small hole pockets. Hence in this region we have $1 \mathrm{D}$ metallic carriers together with small hole pocket. The latter exhibits quantum Hall effect (QHE), which uses the carrier reservoir from 1D carriers. In this region, if the straw-shaped Fermi surface is robust with the field sweep, Shubnikov de Haas effect ( $\mathrm{SdH})$ must be observed. There is an old report of SdH effect observation, claiming $7.2 \cdot 10^{12} \mathrm{~cm}^{-2}$ at $1.1 \mathrm{GPa}$, and $15 \cdot 10^{12} \mathrm{~cm}^{-2}$ at $0.9 \mathrm{GPa}$ with cyclotron mass of $m_{e} / 20$ [24]. We did not confirm SdH effect in our experiments. The reason of the discrepancy can be the sample quality characterized by the sharpness of the transition and the strength of the divergence (resistance increase below $T_{C D W}$ at $p=0$ ). However, it is interesting to note that the cross-sectional area with the assumption of Landau quantum limit at $10 \mathrm{~T}$ gives the value of $4.79 \cdot 10^{12} \mathrm{~cm}^{-2}$ at $1.1 \mathrm{GPa}$ as described in the previous section, which is consistent with the values of Miljak within a factor of two. The reason we did not observe $\mathrm{SdH}$, but instead QHE must be related to the purity of the sample. We speculate that the high purity samples prefer QHE over $\mathrm{SdH}$, with the similar mechanism in (TMTSF) $2 \mathrm{X}$ salt [20], where the nesting vector finds its orientations to the optimum energy minimum for a certain range of magnetic field. In this sense, similar mechanism of QHE in the FISDW of (TMTSF $)_{2} \mathrm{X}$ must have been observed in this probable FICDW of HMTSF-TCNQ.

In region III $\left(B_{z}>10 \mathrm{~T}\right)$, the lowest Landau level of the straw-shaped Fermi pocket is lifted beyond $10 \mathrm{~T}\left(B_{z}\right)$. Then the straw-shaped Fermi pocket disappears, and the electrical conduction from this part disappears above $10 \mathrm{~T}$. Therefore, in this region only one pair of 1D Fermi surface survives. With only this 1D Fermi surface, magnetoresistance of $\rho_{z z}$ in $B_{z}$ diverges if the $v_{z}$-component is cancelled by the general angle in the Lebed-Osada effect.

One (W. Kang) of the present authors has pointed out that Lebed-Osada oscillations disappear when the magnetic field is rotated exactly in the $z-y$ plane [25]. The reason

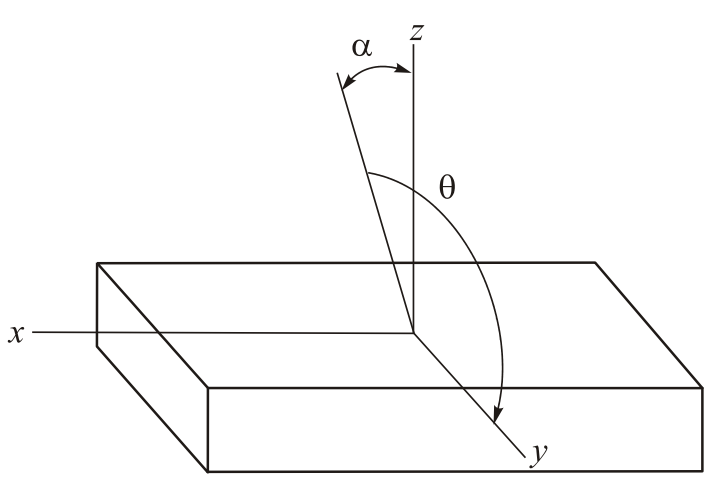

Fig. 9. Actual field sweep is tilted by $\theta$ (15 deg for \#1201, $6 \mathrm{deg}$ for \#1204) in the $z-y$ plane.

that we could observe these oscillations in the $z-y$ plane should be caused by the fact that our rotation is not ideally in the $z-y$ plane since our rotation is of single axis. We verified that the sample is tilted in the pressure cell by $15 \mathrm{deg}$ for \#1201, and $0.6 \mathrm{deg}$. for \#1204 (Fig. 9).

In summary, we examined the magnetic-field-induced phases of HMTSF-TCNQ keeping the pressure at low temperatures at $1.1 \mathrm{GPa}$, where ambient pressure $\mathrm{CDW}$ is almost suppressed. With the result of AMRO, magnetoresistance and the preliminary work on Hall effect, it turned out that transitions are of orbital origin, and ruled by the $z$-component of the magnetic field. Similar to the FISDW in (TMTSF) ${ }_{2} \mathrm{X}$ with QHE, our observation for HMTSF-TCNQ is probably FICDW with QHE. If field-induced phase is CDW, and if the Hall effect is QHE, we can state that the duality (i.e. almost the same scenario except CDW or SDW) between FISDW and FICDW might hold.

Whether or not the field-induced phase is associated with CDW will be verified with NMR, and a precise measurement of Hall effect in the close future.

\section{Acknowledgment}

We are grateful to Professor Denis Jérome for the useful discussion at the early stage of this work.

This work was carried out at the National High Magnetic Field Laboratory at Tallahassee, USA, starting from the preliminary work performed at High Field Laboratory for Superconducting Materials, Institute for Materials Research, Tohoku University (Project No 09H0072, 10H0005, 11H0046).

This work is partially supported by IMR Tohoku Univ. (Project No 12H0022 ), JSPS Inst. Prog. for Young Researcher Overseas Visits "Promoting International Young Researchers in Mathematics and Math. Sci. by OCAMI", and Grant-in-Aid Sci. Res. on Innovative Areas \#23110722 from M. Education, and NSF DMR\#1005293(FSU). W/K was supported by a grant from the National Research Foundation of Korea (NRF), funded by the Korean Government (MSIP) (Nos. 2011-0030902, 2010-00453 and 2008-0061893). Work 
at the NHMFL is supported by the NSF DMR\#0654118, and by the State of Florida.

To achieve this experiment, the assistance of A. Kiswandhi, S. Benjamin, L. Winter of the Brooks group was indispensable. We appreciate Prof. H. Fujiwara of Osaka Prefecture University for the $\mathrm{x}$-ray examination for selecting high quality crystals.

1. D. Jérome, A. Mazaud, M. Ribault, and K. Bechgaard, J. Phys. Lett. (Paris) 41, 95 (1980).

2. P. Chaikin, J. Phys. I 6, 1875 (1996).

3. T.E. Phillips, T.J. Kistenmacher, A.N. Bloch, and D.C. Cowan, J. Chem. Soc. 66, 334 (1976).

4. A.N. Bloch, D.O. Cowan, K. Bechgaard, R.E. Pyle, R.H. Banks, and T.O. Poehler, Phys. Rev. 34, 1561 (1975).

5. C. Weyl, E.M. Engler, K. Bechgaard, D. Jehanno, and S. Etemad, Solid State Commun. 19, 925 (1976).

6. D. Jérome and H.J. Schulz, Adv. Phys. 51, 400 (2002).

7. S. Yasuzuka, K. Murata, T. Arimoto, and R. Kato, J. Phys. Soc. Jpn. 76, 033701 (2007).

8. K. Murata, K. Yokogawa, J.S. Brooks, A. Kismarahardja, E. Steven, M. Kano, Y. Seno, N.R. Tamilselvan, H. Yoshino, T. Sasaki, D. Jérome, P. Senzier, K. Bechgaard, M. Uruichi, and K .Yakushi, J. Phys. Conf. Ser. 215, 012064 (2010).

9. K. Murata, K. Yokogawa, J.S. Brook, A. Kismarahardja, E. Steven, M. Kano, Y. Seno, N.R. Tamilselvan, H. Yoshino, T. Sasaki, D. Jérome, P. Senzier, K. Bechgaard, M. Uruichi, and K. Yakushi, Physica B 405, S111 (2010).

10. K. Murata, K. Yokogawa, K. Kobayashi, K. Masuda, T. Sasaki, Y. Seno, N.R. Tamilselvan, H. Yoshino, J.S. Brooks, D. Jérome, K. Bechgaard, M. Uruichi, K. Yakushi, Y. Nogami, and R. Kato, J. Phys. Soc. Jpn. 79, 103702 (2010).
11. K. Murata, W. Kang, K. Masuda, T. Kuse, T. Sasaki, K. Yokogawa, H. Yoshino, J.S. Brooks, E.S. Choi, A. Kiswandhi, and R. Kato, Physica B 407, 1927 (2012).

12. K. Murata, W. Kang, K. Masuda, Y. Fukumoto, D. Graf, A. Kiswandhi, E.S. Choi, J.S. Brooks, T. Sasaki, K. Yokogawa, H. Yoshino, and R. Kato, J. Low Temp. Phys. 170, 377 (2013).

13. M. Kartsovnik and V.N. Laukhin, J. Phys. I (France) 6, 1753 (1996).

14. A.G. Lebed, Phys. Rev. Lett. 103, 046401 (2009).

15. M. Weger, Solid State Commun. 19, 1149 (1976).

16. K. Murata, H. Yoshino, H.O. Yadav, Y. Honda, and N. Shirakawa, Rev. Sci. Instrum. 68, 2490 (1997).

17. K. Yokogawa, K. Murata, H. Yoshino, and S. Aoyama, Jpn. J. Appl. Phys. 46, 3636 (2007).

18. A.G. Lebed, Pis'ma Zh. Eksp. Teor. Fiz. 43, 137 (1986) [JETP Lett. 43, 174 (1986)].

19. T. Osada, A. Kawasumi, S. Kagoshima, N. Miura, and G. Saito, Phys. Rev. Lett. 66, 1525 (1991).

20. G.M. Danner, W. Kang, and P.M. Chaikin, Phys. Rev. Lett. 72, 3714 (1994).

21. G.M. Danner and P.M. Chaikin, Phys. Rev. Lett. 75, 4690 (1995).

22. B. Morosin, J.E. Schirber, R.L. Greene, and E.M. Engler, Phys. Rev. B 26, 2660 (1982).

23. H. Tanino, K. Kato, M. Tokumoto, H. Anzai, and G. Saito, J. Phys. Soc. Jpn. 54, 2390 (1985).

24. M. Miljak, A. Andrieux, R.H. Friend, G. Malfait, D. Jérome, and K. Bechgaard, Solid State Commun. 26, 969 (1978).

25. W. Kang, T. Osada, Y.J. Jo, and H. Kang, Phys. Rev. Lett., 99, 017002 (2007). 